\title{
Comparison between YM099 and Captopril in Rats with Renal Mass Reduction-Induced Progressive Renal Disease
}

\author{
Takeyuki YATsu, ${ }^{*}$ Motonori AoKI, Wataru UchidA, and Osamu InAGAKI \\ Institute for Drug Discovery Research, Yamanouchi Pharmaceutical Co., Ltd.; 21 Miyukigaoka, Tsukuba, Ibaraki \\ 305-8585, Japan. Received September 29, 2004; accepted November 19, 2004; published online November 24, 2004
}

\begin{abstract}
The effects of the ATP-sensitive potassium $\left(K_{\text {ATP }}\right)$ channel opener YM099, and the angiotensin-converting enzyme (ACE) inhibitor captopril, on the progression of renal disease in rats with surgical renal mass reduction (RMR) were evaluated. Rats were subtotal (5/6) nephrectomized by resection of the renal poles. After 2 weeks of RMR, rats were randomized to three groups and treated for 6 weeks: no treatment $(n=9)$; YM099 at a dose of $0.3 \mathrm{mg} / \mathrm{kg}$ by daily oral administration $(n=9)$; or captopril at a dose of $50 \mathrm{mg} / \mathrm{kg}$ by daily oral administration $(n=9)$. Sham-operated rats were used as normal animals $(n=9)$. In RMR rats with no treatment, proteinuria progressively developed. At 8 weeks after RMR, renal function as assessed by plasma creatinine (Pcr) and blood urea nitrogen (BUN) was impaired. Pharmacological activation of $K_{\text {ATP }}$ channel opening by YM099 showed no beneficial effect on proteinuria and renal functional parameters. On the other hand, pharmacological ACE inhibition by captopril significantly attenuated proteinuria, and tended to inhibit the increases in Pcr and BUN; however, these effects were not statistically significant. The presents study indicates that YM099 exhibits no renoprotection with antiproteinuric effect in rats with progressive renal disease. These findings suggest that activation of $K_{\mathrm{ATP}}$ channel opening may play no role in the retardation of progressive renal disease.
\end{abstract} tion

Key words antiproteinuric effect; $\mathrm{K}_{\text {ATP }}$ channel opener; progressive renal disease; proteinuria; renal mass reduction; renoprotec-

YM099 is an ATP-sensitive potassium $\left(\mathrm{K}_{\mathrm{ATP}}\right)$ channel opener. ${ }^{1,2)}$ Several reports have shown that $K_{\text {ATP }}$ channel openers exhibited beneficial effect on renal hemodynamics and function. ${ }^{3,4)}$ To our knowledge, the effects of the $\mathrm{K}_{\text {ATP }}$ channel openers on the progression of renal disease have not been determined. The renal adaptive response to surgical reduction in nephron number in rats appears close enough to the pathophysiological characteristics of human progressive renal disease. ${ }^{5)}$ In this modesl, an initial adaptive increase in single-nephron glomerular filtration rate and plasma flow associated with glomerular hypertension is followed by progressive renal function deterioration with severe proteinuria and renal histological changes. ${ }^{6-9)}$ The angiotensin-converting enzyme (ACE) inhibitor, captopril, is one of the few drugs to demonstrate renoprotection and an antiproteinuric effect in patients with renal disease. ${ }^{10)}$ Moreover, several experimental studies showed that captopril ( 38 to $42 \mathrm{mg} / \mathrm{kg} / \mathrm{d}$ ) was effective in the $5 / 6$ nephrectomy rat model of progressive renal disease. ${ }^{11,12)}$

Proteinuria is a hallmark of glomerular injury, and evidence has recently emerged that proteinuria is not simply a result of glomerular lesions, but in turn induces tubulointerstitial injury leading to further renal damage. ${ }^{13)}$ Recently, we have indicated that severity of proteinuria positively correlated with plasma creatinine (Pcr), renal hypertrophy, renal histological damage, and systolic blood pressure in rats with renal mass reduction (RMR)-induced progressive renal disease. ${ }^{14)}$ Therefore, prevention of proteinuria might exert a more potent therapeutic effect on the progression of the renal disease than previously believed. It has been already demonstrated that YM099 (0.1, $0.3 \mathrm{mg} / \mathrm{kg}$ p.o.) exerts a dose-dependent and sustained blood pressure lowering effect in spontaneously hypertensive rats. ${ }^{15)}$ Based on these findings, in the present experiment, the effects of YM099 $(0.3 \mathrm{mg} / \mathrm{kg} / \mathrm{d})$ and captopril $(50 \mathrm{mg} / \mathrm{kg} / \mathrm{d})$ on proteinuria were evaluated for up to 8 weeks after RMR. In addition, the effects of YM099 and captopril on renal function as assessed by plasma creatinine (Pcr) and blood urea nitrogen (BUN) were determined at the end of the experiment.

\section{MATERIALS AND METHODS}

Male Sprague-Dawley rats (8 weeks old) were obtained from Japan SLC (Shizuoka, Japan). All animals were housed in communal cages and maintained on a $12 \mathrm{~h} \mathrm{light/dark} \mathrm{cycle}$ with food and water available ad libitum. All experimental procedures involving animals conformed to the regulations of the Animal Ethical Committee of Yamanouchi Pharmaceutical Co., Ltd. and "The Guide for the Care and Use of Laboratory Animals" (U.S. Department of Health and Human Services, 1985 NIH Publication No. 85-23).

YM099, 2-(7,8-dihydro-6,6-dimethyl-6H-oxozino(2,3-e)$(2,1,3)$ benzoxadiazol-8-yl)pyridine $N$-oxide, was synthesized by Yamanouchi Pharmaceutical Co., Ltd. (Tokyo, Japan). Captopril was purchased from the Sigma Chemical Company (MO, U.S.A.). YM099 and captopril were suspended in 0.5\% methylcellulose in water and administered in a volume of $5 \mathrm{ml} / \mathrm{kg}$ by once daily gastric gavage.

RMR was performed by the surgical excision method, as previously described. ${ }^{14)}$ All surgical procedures were carried out under anesthesia with ether. The left kidney was exposed through a left retroperitoneal incision and decapsulated, leaving the adrenal gland intact. The upper and lower poles (two thirds of the left kiney) were resected, and the remnant kidney was allowed to recover for 1 week. Then the remaining right kidney was removed through a right retroperitoneal incision after ligation of the right renal artery, vein, and ureter. Sham-operated rats underwent the same anesthesia and laparotomy without destruction of renal tissue.

Twenty seven rats with RMR and 9 sham-operated rats were used. Two, 4, 6 and 8 weeks after surgery, rats were housed individually in metabolic cage for $24 \mathrm{~h}$. At 2 weeks 
after RMR, the animals were randomly divided into the following three groups: no treatment $(n=9)$; YM099 at a dose of $0.3 \mathrm{mg} / \mathrm{kg}$ by daily oral administration $(n=9)$; or captopril at a dose of $50 \mathrm{mg} / \mathrm{kg}$ by daily oral administration $(n=9)$. Sham-operated rats were used as normal animals $(n=9)$.

For urine parameters measurements, each rat was separately housed in a metabolic cage (Natsume Manufacturing, Tokyo, Japan). Protein concentration in urine samples was determined by the sulphosalicyclic method. ${ }^{16)}$ At the end of the experiment, animals were anesthetized with ether, and blood was taken from the abdominal aorta for measurement of plasma creatinine (Pcr) and blood urea nitrogen (BUN). The plasma was separated by centrifugation. Pcr and BUN were measured with an automatic analyzer (Model 7250, HITACHI, Japan).

Data are expressed as mean \pm S.E.M. of nine experiments. Data were analyzed by one-way analysis of variance followed by Tukey's multiple comparison test. $p<0.05$ was considered statistically significant.

\section{RESULTS}

There were no differences in increases in body weight among the three experimental groups for 8 weeks following RMR (Fig. 1). Changes in urinary protein excretion during the experiment period are shown in Fig. 1. At 2 weeks after RMR, there were no differences in urinary protein excretion among the three experimental groups. Following RMR, rats with no treatment manifested progressively increasing pro-

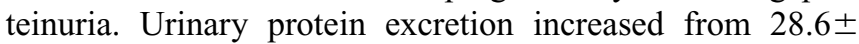
$3.9 \mathrm{mg} / \mathrm{d}$ ( 2 weeks) to $190.6 \pm 26.3 \mathrm{mg} / \mathrm{d}$ ( 8 weeks). Treatment with YM099 for 6 weeks did not lead to blunting of the increases in proteinuria. On the other hand, treatment with captopril for 6 weeks blunted the proteinuria throughout the experiment. Urinary protein excretion in RMR rats with captopril was significantly less than that in RMR rat with no treatment and YM099 at all time points except for 6 weeks. Urinary protein excretion in RMR rats was significantly higher than that in sham-operated rats during the entire experiments.

Renal function as assessed by Pcr and BUN at the end of the experiment ( 8 weeks) is shown in Fig. 2. Pcr and BUN in RMR rats with no treatment significantly elevated as compared with those in sham-operated rats. Pcr and BUN in RMR rats with YM099 were not statistically different from that observed in RMR rats with no treatment. Captopril tended to inhibit the increases in Pcr and BUN; however, this effect was not statistically significant.

\section{DISCUSSION}

This is the first study to assess the effect of long-term activation of $\mathrm{K}_{\mathrm{ATP}}$ channel opening on proteinuria and renal function in rats with progressive renal disease. In the present study we have demonstrated that the $\mathrm{K}_{\mathrm{ATP}}$ channel opener YM099 exhibited no renoprotection with antiproteinuric effect. On the other hand, the ACE inhibitor captopril attenuated proteinuria and the elevation of Pcr and BUN. These data would be almost consistent with previous reports that AEC inhibitors showed renoprotection with antiproteinuric effect in rats with progressive renal disease. ${ }^{11,14)}$ In this study,
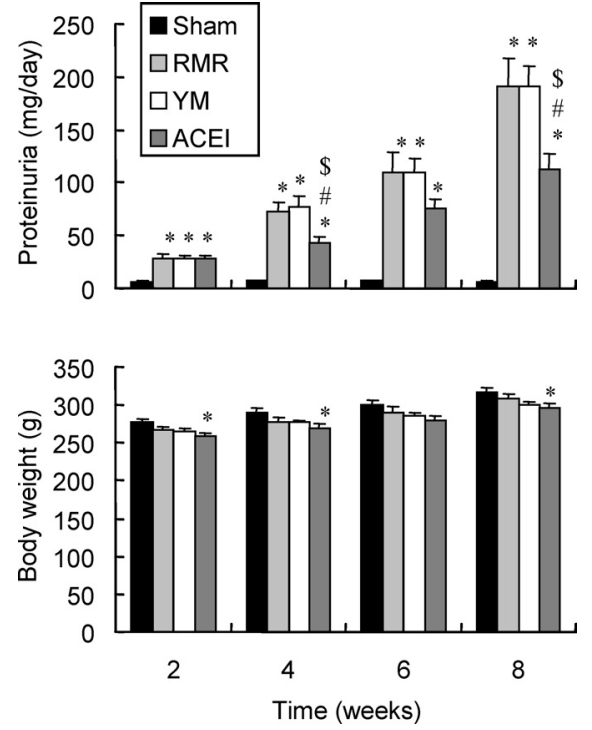

Fig. 1. Time Course of Urinary Protein Excretion (Upper Panel) and Body Weight (Lower Panel) in Renal Mass Reduction Rats with No Treatment (RMR), YM099 Treatment (YM) and Captopril Treatment (ACEI), or Sham-Operated Rats (Sham)

Values are mean \pm S.E.M. of 9 animals per group. $* p<0.01 v s$. Sham; ${ }^{*} p<0.05 v$ s. $\mathrm{RMR} ; \$ p<0.05$ vs. YM.
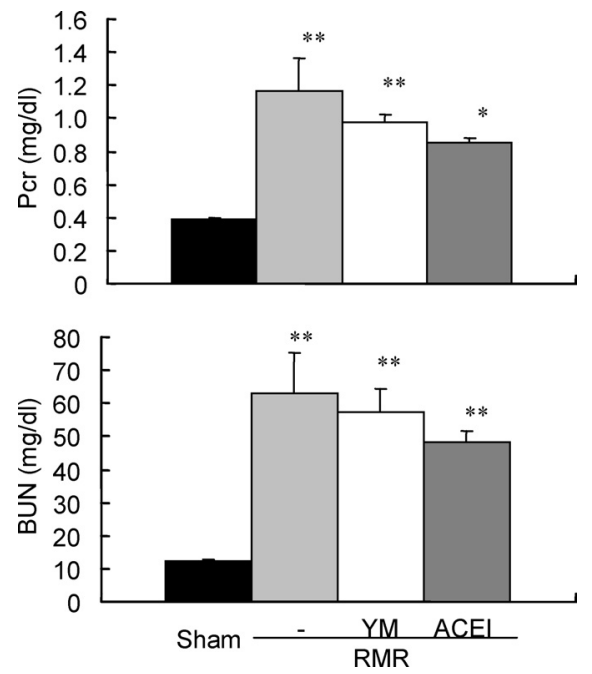

Fig. 2. Plasma Creatinine (Pcr; Upper Panel) and Blood Urea Nitrogen (BUN; Lower Panel) in Renal Mass Reduction Rats with No Treatment (RMR), YM099 Treatment (YM) and Captopril Treatment (ACEI), or Sham-Operated Rats at 8 Weeks after Surgery

Values are mean \pm S.E.M. of 9 animals per group. $* p<0.05$, $* * p<0.01 v s$. Sham.

the captopril effect on renal function was not statistically significant probably for three reasons that 1) relatively small number of cases (there is no statistical power), 2) the relatively short follow up (8 weeks), 3) the initial apparent negative effect of ACEIs on renal function, with a longer time being necessary to demonstrate a beneficial effect of the drug on the rate of progression. Recent studies have demonstrated that proteinuria per se plays an important role in renal failure progression. ${ }^{17-19)}$ Reduction of proteinuria as a means of slowing loss of renal function is becoming recognized as important in patients with chronic renal disease. ${ }^{20)}$ The present study revealed that YM099 did not attenuate proteinuria and 
the increases in Pcr and BUN. These results are consistent with a recent report that the severity of proteinuria positively correlated with impaired renal function as assessed by Pcr in rats with RMR-induced progressive renal disease. ${ }^{14)}$

Surgical renal mass reduction rats, which is the commonly used experimental animal model of chronic renal failure appear close enough to the pathophysiological characteristics of human progressive renal disease. ${ }^{6,8,9)}$ We used this model to directly compare the potential renoprotective effects of two classes of agents: $\mathrm{K}_{\mathrm{ATP}}$ channel openers and ACE inhibitors. We administered the two classes of agents by gastric gavage once daily, assuring accurate drug delivery when compared with providing the drugs in food or drinking water. The effects of $\mathrm{K}_{\text {ATP }}$ channel opening on proteinuria were markedly different from those observed with ACE inhibition. At 4, 6 and 8 weeks after RMR, rat receiving YM099 had a urinary protein excretion equivalent to that observed in untreated rats. This disparate effect on proteinuria of ACE inhibitor and $\mathrm{K}_{\text {ATP }}$ channel opener may be explained by following reasons. The renal hemodynamic profiles of these agents are distinctly different. In particular, although it is clear that ACE inhibitors reduce glomerular hypertension, ${ }^{5,7,21)} \mathrm{K}_{\text {ATP }}$ channel opener may not lower glomerular hypertension, because of a concomitant reduction in preglomerular resistances. This consideration is supported by previous reports that increase in renal blood flow by $\mathrm{K}_{\text {ATP }}$ channel openers was accompanied by increase in glomerular filtration rate. ${ }^{3,15)}$ In addition, it has been reported that $\mathrm{K}_{\text {ATP }}$ channel opener stimulated renin release, ${ }^{3)}$ leading to increase in postglomerular resistances. It is possible that these disparate effects on glomerular hypertension underlie the different effects on urinary protein excretion; however, without direct measurements of glomerular hemodynamics these proposed mechanisms must remain speculative.

Recent our study indicated that the severity of proteinuria positively correlated with the severity of renal histological injury (renal fibrosis) and renal hypertrophy, and ACE inhibitor limited renal hypertrophy and histological changes, suggesting that the antiproteinuric efficacy of specific drugs is crucial to their long-term renoprotecitive potential. ${ }^{14)}$ In clinical studies of parallel design, the treatment group with more effective reduction of proteinuria invariably shows more effective renoprotection. ${ }^{22}$ Based on these finding, although we did not evaluate renal morphology in this study, $\mathrm{K}_{\text {ATP }}$ channel opener may provide a lack of beneficial effect on renal fibrosis.

In conclusion, $\mathrm{K}_{\text {ATP }}$ channel opener had no effect on proteinuria and renal function in rats with RMR-induced progressive renal disease. However, ACE inhibitor reduced pro- teinuria and the elevation of Pcr and BUN. The current study dose not support a primary role for activation of $\mathrm{K}_{\text {ATP }}$ channel opening in the retardation of progressive renal disease.

Aknowledgments The authors acknowledge Drs. Toichi Takenaka, Isao Yanagisawa, Takashi Fujikura, Gensei Kon, Masaharu Asano and Nobuyuki Yamamoto (Yamanouchi Pharmaceutical Co., Ltd.) for their valuable comments and continuing encouragement.

\section{REFERENCES}

1) Uchida W., Hirano Y., Taguchi T., Masuda N., Shirai Y., Satoh N., Takenaka T., Eur. J. Pharmacol., 264, 285-293 (1994).

2) Uchida W., Hirano Y., Shirai Y., Taguchi T., Masuda N., Shibasaki K., Hirano S., Matsumoto Y., Tsuzuki R., Yanagisawa I., Arch. Int. Pharmacodyn., 327, 330-343 (1994).

3) Hayashi K., Matsumura Y., Yoshida Y., Ohyama T., Hisaki K., Suzuki Y., Morimoto S., J. Pharmacol. Exp. Ther, 252, 1240-1246 (1990).

4) Tamaki T., Hasui K., Shoji T., Fukui K., Iwao H., Abe Y., J. Cardiovasc. Pharmacol., 17, 305-309 (1991).

5) Brenner B. M., Lawler E. V., Mackenzie H. S., Kidney Int., 49, 1774 1777 (1996).

6) Shimamura T., Morrison A. B., Am. J. Pathol., 79, 95-106 (1975).

7) Anderson S., Meyer T. W., Rennke H. G., Brenner B. M., J. Clin. Invest. 76, 612-619 (1985).

8) Noda M., Matsuo T., Fukuda R., Ohta M., Nagano H., Shibouta Y., Naka T., Nishikawa K., Imura Y., Kidney Int., 56, 898-909 (1999).

9) Badid C., Vincent M., McGregor B., Melin M., Hadj-Aissa A., Veysseyre C., Hartmann D. J., Desmouliere A., Laville M., Kidney Int., 58, 51-61 (2000).

10) Lewis E. J., Hunsicker L. G., Rohde R. D., N. Engl. J. Med., 329 , 1456-1462 (1993).

11) Brooks D. P., Contino L. C., Short B. G., Gowan C., Trizna W., Edwards R. M., J. Pharmacol. Exp. Ther, 274, 1222-1227 (1995).

12) Brooks D. P., Short B. G., Cyronak M. J., Contino L. C., DiCristo M., Wang Y. X., Ruffolo R. R., Jr., Br. J. Pharmacol., 109, 581-586 (1993).

13) Remuzzi G., Bertani T., N. Engl. J. Med., 339, 1448-1456 (1998).

14) Yatsu T., Sanagi M., Fujimori A., Tomura Y., Hayashi K., Tanahashi M., Inagaki O., Pharmacol. Res., 47, 243-252 (2003).

15) Masuda N., Uchida W., Arai Y., Taguchi T., Tsuzuki R., Inagaki O., Jpn. J. Pharmacol., 61 (Suppl. 1), 209P (1993).

16) Davidsohn L., Henry J. B., "Clinical Diagnosis by Laboratory Methods," 14th ed., WB Saunders, Philadelphia, 1969.

17) Remuzzi G., Ruggenenti P., Benigni A., Kidney Int., 51, 2-15 (1997).

18) Mackenzie H. S., Brenner B. M., Am. J. Kidney Dis., 31, 161-170 (1998).

19) Gomez-Garre D., Largo R., Tejera N., Fortes J., Manzarbeitia F., Egido J., Hypertension, 37, 1171-1178 (2001).

20) Locatelli F., Vecchio L. D., Pozzoni P., Nephrol. Dial. Transplant., 17 (Suppl. 11), 2-7 (2002).

21) Hostetter T. H., Olson J. L., Rennke H. G., Venkatachalam M. A., Brenner B. M., Am. J. Physiol., 241, F85-F93 (1981).

22) Anonymous., Lancet, 349, 1857-1863 (1997). 